\title{
Effect of quercetin on altered vascular reactivity in aortas isolated from streptozotocin-induced diabetic rats
}

\author{
Machha Ajay ${ }^{\mathrm{a}}$, Francis I. Achike ${ }^{\mathrm{b}}$, Ali Mohd Mustafa ${ }^{\mathrm{a}}$, \\ Mohd Rais Mustafa ${ }^{\text {a,* }}$

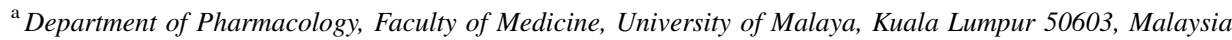 \\ ${ }^{\mathrm{b}}$ International Medical University, Kuala Lumpur 57000, Malaysia
}

Received 25 August 2005; accepted 21 November 2005

Available online 27 December 2005

\begin{abstract}
The present work examined ex vivo the acute effect of quercetin on diabetic rat aortic ring reactivity in response to endotheliumdependent (acetylcholine, ACh) and endothelium-independent (sodium nitroprusside, SNP) relaxants, and to the $\alpha_{1}$-adrenergic agonist phenylephrine (PE). Responses were compared to those of aortic rings from age- and sex-matched euglycemic rats. Compared to euglycemic rat aortic rings, diabetic rings showed less relaxation in response to ACh and SNP, and greater contraction in response to PE. Pretreatment with quercetin $(10 \mu \mathrm{M}, 20 \mathrm{~min})$ increased ACh-induced relaxation and decreased PE-induced contraction in diabetic, but did not affect euglycemic rat aortic ring responses. Following pretreatment with the nitric oxide synthase inhibitor N $\omega$-nitro-L-arginine methyl ester (L-NAME, $10 \mu \mathrm{M}$ ), quercetin reduced PE-induced contractions in both aortic ring types, although L-NAME attenuated the reduction in the diabetic rings. Quercetin did not alter SNP vasodilatory effects in either ring type compared to their respective controls. These findings indicate that quercetin acutely improved vascular responsiveness in blood vessels from diabetic rats, and that these effects were mediated, at least in part, by enhanced endothelial nitric oxide bioavailability. These effects of quercetin suggest the possible beneficial effects of quercetin in vivo in experimental diabetes and possibly in other cardiovascular diseases.
\end{abstract}

(C) 2005 Elsevier Ireland Ltd. All rights reserved.

Keywords: Quercetin; Flavonoids; Free radicals; Nitric oxide; Endothelium; Diabetes

\section{Introduction}

Endothelial cells produce several biologically active substances that play key roles in local regulation of blood flow, blood pressure and vascular tone, with one of the most important being nitric oxide (NO) [1,2]. Physiological functions of endothelial cells are altered in certain cardiovascular disease states, including diabetes [3-5]. Diabetes is characterized by increased

\footnotetext{
* Corresponding author. Tel.: +60 3 79674952; fax: +60 379674791 .

E-mail address: rais@um.edu.my (M.R. Mustafa).
}

oxidant stress which is believed to play an important role in the development of vascular complications such as reduced endothelium-dependent relaxation (EDR) and enhanced receptor-mediated contractions associated with diabetes [4-6]. Indeed, recent studies have shown that short-term in vitro and long-term in vivo antioxidant interventions improved endothelial function in diabetes [6,7], suggesting a pathological role for oxygen-derived free radicals in the impaired vascular responses in diabetes.

Flavonoid-rich diets are reported to have beneficial effects in cardiovascular disease states associated with overproduction of reactive oxygen species. For example, the Rotterdam study showed an inverse association 
between flavonoid intake and occurrence of myocardial infarction [8]. These protective effects of flavonoids are chiefly ascribed to their antioxidant and vasodilator actions $[9,10]$. Quercetin, the most commonly found flavonoid in the human diet, has been shown to exert potent free radical scavenging and antioxidant actions, to cause vasodilatation in isolated vascular preparations, and to elicit blood glucose-lowering (anti-diabetic) effects in experimental diabetes [11-15]. In addition, we and others have demonstrated the endothelial protective effects of chronic quercetin in experimental hypertension $[16,17]$. However, to our knowledge there is no information available about the effects of quercetin on impaired vascular reactivity of diabetic arteries.

Using aortic rings isolated from streptozotocin (STZ)-induced diabetic rat, in the present work, therefore, we investigated whether quercetin could acutely modulate relaxant and contractile responses in diabetic blood vessels.

\section{Materials and methods}

\subsection{Drugs and chemicals}

Quercetin, phenylephrine-HCl (PE), acetylcholine chloride $(\mathrm{ACh})$, indomethacin, $\omega$-nitro-L-arginine methyl ester (LNAME), and streptozotocin (STZ) were purchased from Sigma Chemicals Company (St. Louis, MO, USA). Sodium nitroprusside (SNP) and Krebs salts were purchased from BDH Limited and BDH Laboratory Supplies (Poole, UK), respectively. Except for quercetin, all the other drug solutions were prepared fresh on the day of experiment by dissolving weighed amounts of respective drugs in distilled water. Quercetin stock solution $(10 \mathrm{mM})$ was prepared in $5 \%(\mathrm{v} / \mathrm{v})$ dimethyl sulfoxide (DMSO). The final concentrations were prepared by serial dilutions with distilled water and the final concentration of DMSO was adjusted to less than $0.05 \%$ (v/v).

\subsection{Experimental animals}

The animals were obtained from the University of Malaya animal unit, and all the experimental procedures were performed in accordance with guidelines issued by the University of Malaya Animal Experimentation Ethics Committee. Male Wistar-Kyoto (WKY) rats, aged about $12-13$ weeks, were divided randomly into two groups, each comprising of 15 animals. The rats were maintained under controlled room conditions and supplied with standard rat chow and tap water ad libitium. After 1 week of acclimatization period, diabetes was induced in one group of rats by a single intraperitoneal dose $(75 \mathrm{mg} / \mathrm{kg})$ of STZ dissolved in cold normal saline. Plasmatic glycemia was examined 3 days after diabetes induction and the rats were considered as diabetics only if their blood glucose level exceeds $17 \mathrm{mmol} / \mathrm{l}$. The rats were then maintained at the specified conditions for 8 weeks prior to being sacrificed at the age of 20-21 weeks.

\subsection{Measurement of ex vivo vascular function}

The diabetic and control euglycemic rats were anaesthetized with pentobarbital $(60 \mathrm{mg} / \mathrm{kg}$, IP). The descending thoracic aorta was excised by midline incision, cleaned of fat and connective tissues, with care taken not to stretch the vessel excessively or to disturb the luminal surface of the rings, to ensure the integrity of the endothelium. The aorta was then cut into small rings (3-5 $\mathrm{mm}$ in width) and suspended between two wire stirrups in a jacketed organ bath containing $5 \mathrm{ml}$ of normal Krebs physiological solution (KPS) of the following composition (mM): $\mathrm{NaCl} 118.2, \mathrm{KCl} 4.7, \mathrm{CaCl}_{2} \cdot 2 \mathrm{H}_{2} \mathrm{O} 2.5$, $\mathrm{KH}_{2} \mathrm{PO}_{4} 1.2, \mathrm{MgCl}_{2}$ 1.2, glucose 11.7, $\mathrm{NaHCO}_{3}$ 25.0, and EDTA 0.026 . The bath solution was maintained at $37^{\circ} \mathrm{C}$ and oxygenated continuously with a mixture of $95 \%$ oxygen and $5 \%$ carbon dioxide. The rings were then progressively stretched to a preload tension of $1 \mathrm{~g}$ and allowed to equilibrate for $45 \mathrm{~min}$. During this period the bath solution was replaced every $15 \mathrm{~min}$. Following the equilibration period, aortic rings were exposed twice (each for $5 \mathrm{~min}$ ) to isotonic potassium chloride solution (high $\mathrm{K}^{+}, 80 \mathrm{mM}$ ). After washout of responses to high $\mathrm{K}^{+}$, the relaxation responses to $\mathrm{ACh}$ and SNP or contractile responses to PE were recorded in the aortic rings. Indomethacin $(10 \mu \mathrm{M})$ was added to the bath for all the experiments to inhibit the synthesis of endoperoxides including the prostaglandins [7]. Aortic rings from diabetic and agematched euglycemic rats were incubated for $20 \mathrm{~min}$ in KPS to which was added quercetin $(10 \mu \mathrm{M})$ or its vehicle (DMSO). Preliminary studies showed that quercetin or its vehicle did not modify basal tension of aortic rings from both euglycemic and diabetic rats during the time course of incubation before addition of agonists. The choice of $10 \mu \mathrm{M}$ quercetin in this study is based on previous studies in which this concentration was found to induce endothelium and nitric oxide (NO)dependent (against PE-induced contraction) and endothelium-independent (against high $\mathrm{K}^{+}$-induced contraction) relaxation in isolated euglycemic [13] and diabetic [15] rat aortas.

\subsubsection{Relaxations to ACh and SNP}

The relaxations to ACh and SNP were examined in PEprecontracted aortic rings. The rings were exposed to single concentration of PE $(1 \mu \mathrm{M})$ and at the peak of the contraction the relaxations to cumulatively increasing concentrations of ACh $(0.1 \mathrm{nM}-10 \mu \mathrm{M})$ or SNP $(10 \mathrm{pM}-1 \mu \mathrm{M})$ were recorded at $3 \mathrm{~min}$ intervals.

\subsubsection{Contractile responses to $P E$}

The responses of the aortic rings to cumulatively increasing concentrations of PE $(0.1 \mathrm{nM}-10 \mu \mathrm{M})$ were recorded at intervals of $3 \mathrm{~min}$. The protocol was repeated with a separate set of diabetic and euglycemic aortic rings which were exposed to the nitric oxide synthase inhibitor, L-NAME $(10 \mu \mathrm{M})$. 


\subsection{Calculations and statistical analysis}

Relaxation responses to cumulative concentrations of $\mathrm{ACh}$ and SNP were calculated as percentage inhibition of PEinduced maximal contraction. Concentration-dependent contractile responses to PE were recorded as percentage of the maximum contraction obtained following tissue stimulation with high $\mathrm{K}^{+}$. All the results were given as mean \pm standard error of the mean (S.E.M.). The concentration-response curves for each experimental condition was plotted and from it was deduced the maximum agonist-induced response and the $\mathrm{pEC}_{50}$ (negative logarithm of median concentration) values. The differences in responses among the different groups were analyzed for statistical significance using twotailed Student's $t$-test for unpaired observations and one-way analysis of variance (ANOVA, Prism version 2.0, Graphpad
Software, USA) for dose-response curves. In all cases the differences were considered significant only if the $p$ value was less than 0.05 .

\section{Results}

\subsection{ACh- and SNP-induced relaxation}

We examined ACh-induced relaxation in isolated aortic rings from euglycemic and diabetic rats in the presence of vehicle or quercetin (Fig. 1). Euglycemic aortic rings showed a dose-dependent relaxation in response to $\mathrm{ACh}$, with a $\mathrm{pEC}_{50}$ value of 7.75 and $\mathrm{a}$ maximum relaxation $\left(R_{\max }\right)$ of $87 \%$ in terms of the reduction in the peak PE-induced contraction (Table 1).

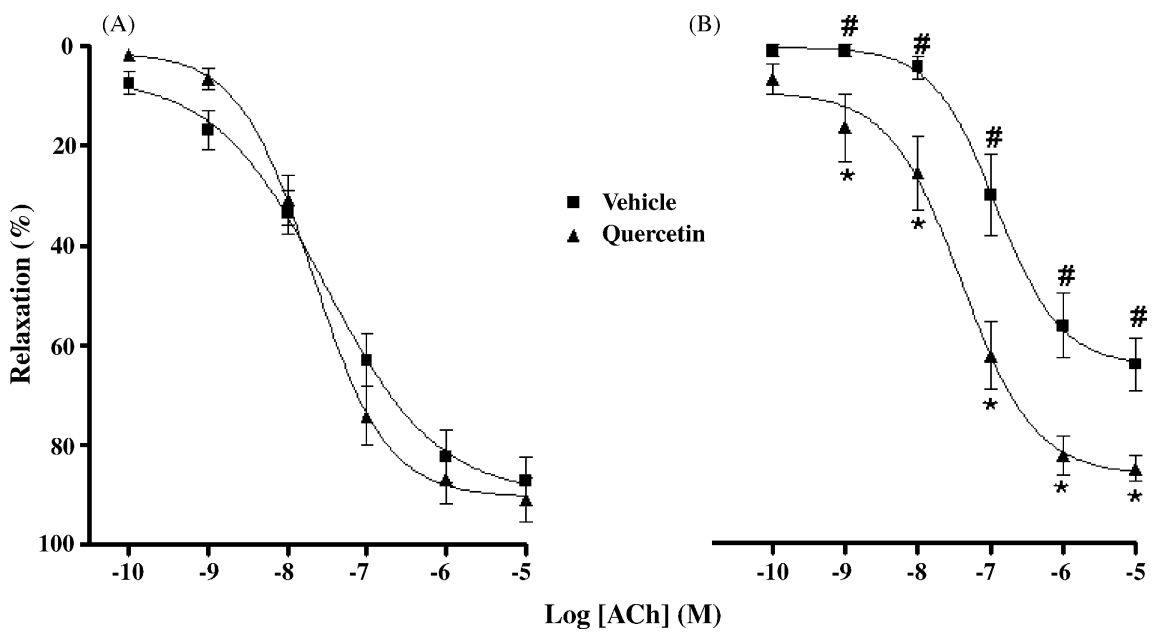

Fig. 1. Effects of quercetin $(10 \mu \mathrm{M})$ on endothelium-dependent relaxation responses to acetylcholine (ACh) in aortic rings of (A) euglycemic and (B) diabetic rats. Symbols represent mean \pm S.E.M. $(n=6$ or 7$) .{ }^{*} p<0.05$ for diabetic aorta vs. corresponding euglycemic aorta; ${ }^{*} p<0.05$ for quercetin vs. vehicle pretreated diabetic aorta.

Table 1

Effects of quercetin on vascular reactivity of aortic rings of euglycemic and STZ-induced diabetic rats

\begin{tabular}{|c|c|c|c|c|c|c|c|}
\hline \multirow[t]{2}{*}{ Rats } & \multirow[t]{2}{*}{ Treatment group } & \multicolumn{2}{|c|}{ Phenylephrine (PE) } & \multicolumn{2}{|c|}{ Acetylcholine (ACh) } & \multicolumn{2}{|c|}{ Sodium nitroprusside (SNP) } \\
\hline & & $\begin{array}{l}\text { Maximum } \\
\text { contraction (\%) }\end{array}$ & $\mathrm{pEC}_{50}$ & $\begin{array}{l}\text { Maximum } \\
\text { relaxation }(\%)\end{array}$ & $\mathrm{pEC}_{50}$ & $\begin{array}{l}\text { Maximum } \\
\text { relaxation }(\%)\end{array}$ & $\mathrm{pEC}_{50}$ \\
\hline \multirow[t]{4}{*}{ Euglycemic } & Vehicle & $126.2 \pm 8.0$ & $7.07 \pm 0.1$ & $86.9 \pm 4.8$ & $7.75 \pm 0.2$ & $104.0 \pm 1.0$ & $7.68 \pm 0.2$ \\
\hline & Quercetin & $136.6 \pm 5.5$ & $6.84 \pm 0.09$ & $91.0 \pm 4.3$ & $7.70 \pm 0.1$ & $104.8 \pm 1.7$ & $7.98 \pm 0.1$ \\
\hline & L-NAME + vehicle & $178.8 \pm 5.3^{\mathrm{a}}$ & $9.02 \pm 1.5$ & ND & ND & ND & ND \\
\hline & L-NAME + quercetin & $151.3 \pm 6.7^{\mathrm{b}}$ & $8.68 \pm 1.6$ & ND & ND & ND & ND \\
\hline \multirow[t]{4}{*}{ Diabetic } & Vehicle & $148.9 \pm 9.0^{\mathrm{a}}$ & $7.17 \pm 0.1$ & $64.1 \pm 5.1^{\mathrm{a}}$ & $6.91 \pm 0.2$ & $95.84 \pm 1.4^{\mathrm{a}}$ & $7.30 \pm 0.4$ \\
\hline & Quercetin & $80.0 \pm 10.8^{\mathrm{a}, \mathrm{c}}$ & $6.99 \pm 0.2$ & $85.0 \pm 2.6$ & $7.39 \pm 0.2$ & $97.61 \pm 2.4$ & $7.46 \pm 0.4$ \\
\hline & L-NAME + vehicle & $176.9 \pm 10.4^{\mathrm{c}}$ & $7.27 \pm 0.1$ & ND & ND & ND & ND \\
\hline & L-NAME + quercetin & $126.8 \pm 7.1^{\mathrm{b}}$ & $7.12 \pm 0.2$ & ND & ND & ND & ND \\
\hline
\end{tabular}

The aortic rings were pretreated with quercetin $(10 \mu \mathrm{M})$ or vehicle (DMSO). ND: not determined.

${ }^{\mathrm{a}} p<0.05$ vs. vehicle pretreated euglycemic aortas.

${ }^{\mathrm{b}} p<0.05$ vs. corresponding L-NAME plus vehicle pretreated aortas.

c $p<0.05$ vs. vehicle pretreated diabetic aortas. 

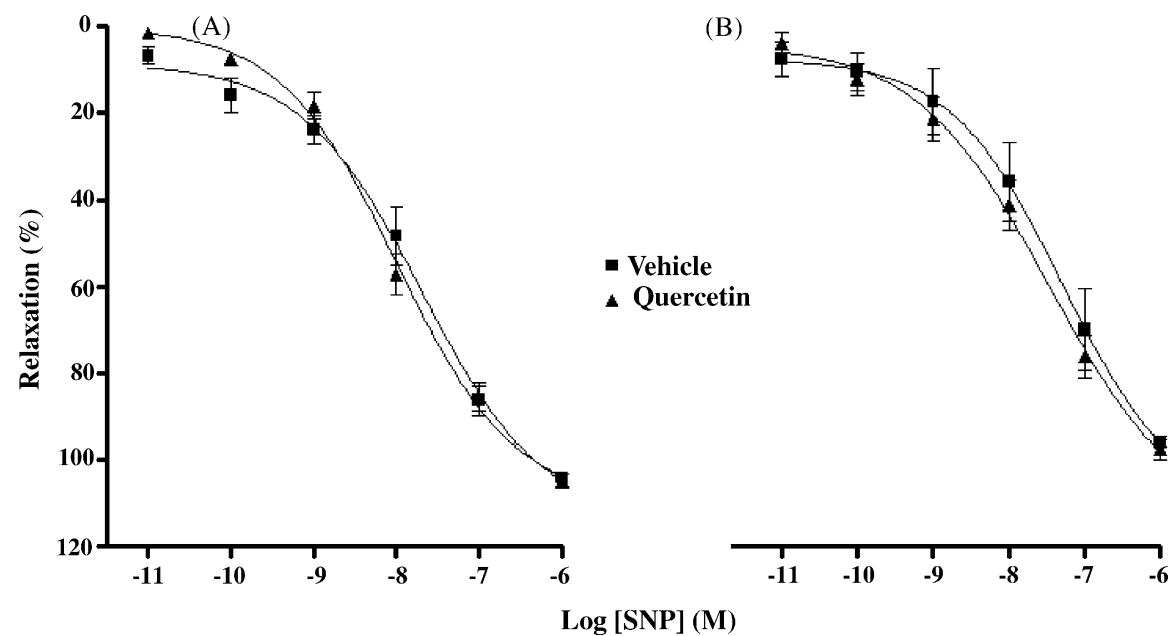

Fig. 2. Effects of quercetin $(10 \mu \mathrm{M})$ on endothelium-independent relaxation responses to sodium nitroprusside (SNP) in aortic rings of (A) euglycemic and (B) diabetic rats. Symbols represent mean \pm S.E.M. $(n=6)$.

Quercetin pre-treatment had no significant effect on the vasorelaxant responses to $\mathrm{ACh} \quad\left(\mathrm{pEC}_{50}=7.70\right.$, $R_{\max }=91 \%$ ) (Fig. 1A). In diabetic aortic rings, the $R_{\max }$ for the ACh response was markedly reduced to $64 \%$ and the curve shifted to the right compared to the euglycemic aortic rings curve (Fig. 1). Quercetin pretreatment of diabetic rings improved the relaxant response to ACh and cause a leftward shift of the doseresponse curve compared to vehicle pretreated aortic rings (Fig. 1B). This improvement in the vasorelaxant response of the diabetic rings to ACh was similar to that for euglycemic aortic rings.

Fig. 2 shows the result of relaxations induced by SNP in aortic rings from the various experimental groups. The relaxation curves for SNP for the various groups were similar, other than a significant reduction in the peak relaxation recorded for the control diabetic rings compared to the equivalent euglycemic aortic rings (Table 1).

\subsection{PE-induced contraction}

We examined aortic ring contractions in response to increasing concentrations of PE (Fig. 3). Euglycemic aortic rings showed concentration-dependent contraction with a $\mathrm{pEC}_{50}$ value of 7.07 and a maximum contractile response $\left(C_{\max }\right)$ of $126 \%$ of the high $\mathrm{K}^{+}$-induced contraction (Table 1). Quercetin pretreatment had little effect on these contractile responses $\left(C_{\max }=136 \%, \mathrm{pEC}_{50}=6.84\right)$. In diabetic

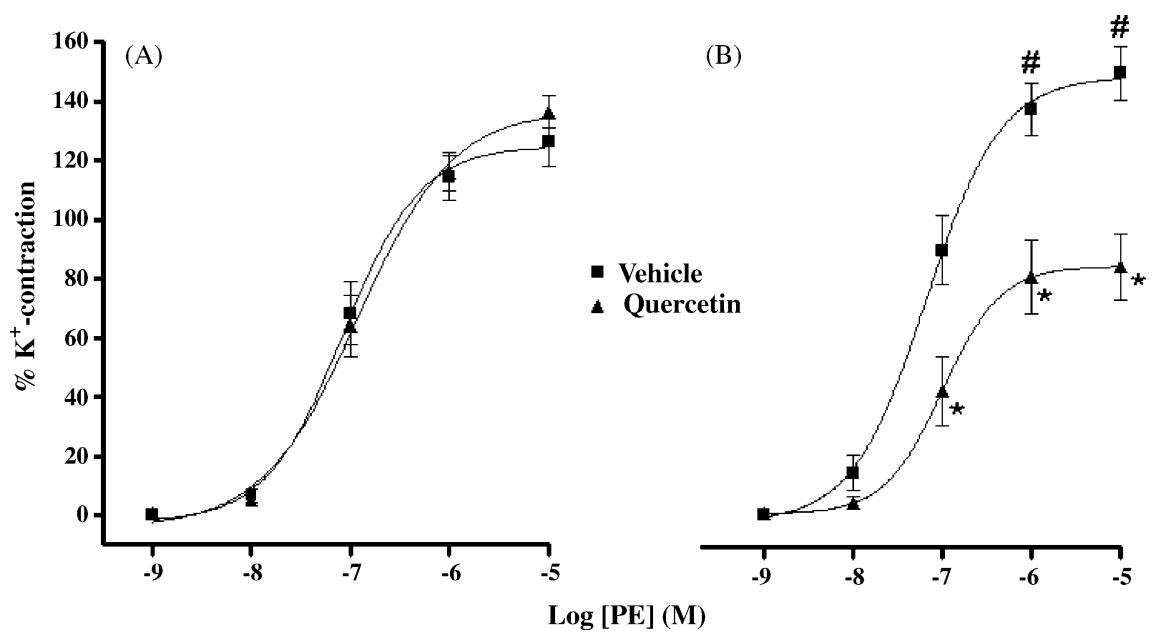

Fig. 3. Effects of quercetin $(10 \mu \mathrm{M})$ on contractile responses to phenylephrine (PE) in aortic rings of (A) euglycemic and (B) diabetic rats. Symbols represent mean \pm S.E.M. $(n=6$ or 7$) .{ }^{*} p<0.05$ for diabetic aorta vs. corresponding euglycemic aorta; ${ }^{*} p<0.05$ for quercetin vs. vehicle pretreated diabetic aorta. 


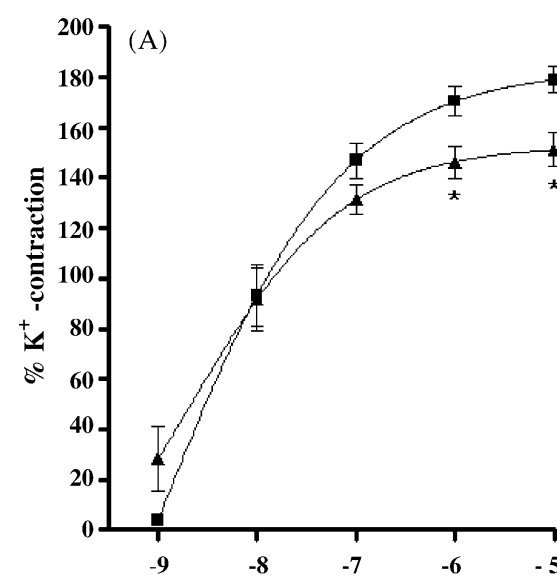

(B)

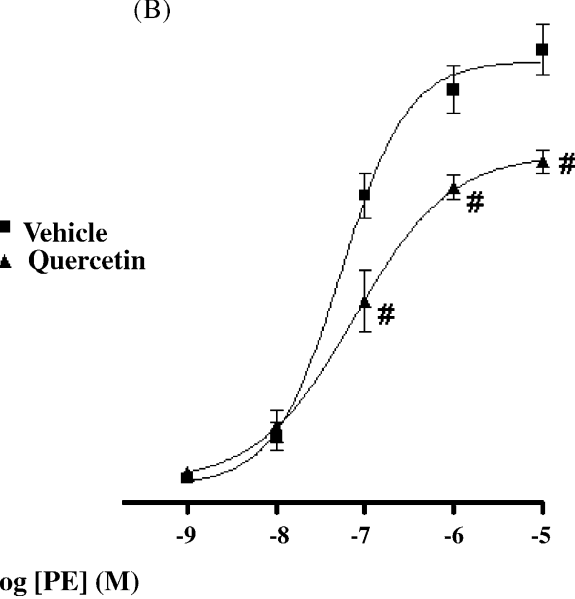

Fig. 4. Effects of quercetin $(10 \mu \mathrm{M})$ on contractile responses to phenylephrine (PE) in the presence of L-NAME in aortic rings of (A) euglycemic and (B) diabetic rats. Symbols represent mean \pm S.E.M. $(n=6$ or 7$) .{ }^{*} p<0.05$ for quercetin vs. vehicle pretreated euglycemic aorta; ${ }^{\#} p<0.05$ for quercetin vs. vehicle pretreated diabetic aorta.

aortic rings, addition of $\mathrm{PE}$ resulted in contraction with a $\mathrm{pEC}_{50}$ value of 7.17 and a higher $C_{\max }$ response of $149 \%$ compared to euglycemic rings. Pretreatment of the diabetic rings with quercetin markedly reduced the responses to $\mathrm{PE}\left(C_{\max }=84 \%, \mathrm{pEC}_{50}\right.$ value of 6.99).

To ascertain the involvement of nitric oxide in the response to quercetin, we examined the effect of the endothelial nitric oxide synthase inhibitor, L-NAME. Compared to their respective vehicle incubated control rings, the addition of L-NAME to the incubation mixture increased the $C_{\max }$ response to PE (Fig. 4). For both euglycemic and diabetic rings, the addition of quercetin to the incubation mixture containing L-NAME caused the contractile response curves for PE to shift downwards of those generated from L-NAME alone incubations. However, the percentage depression of the $C_{\max }$ response to $\mathrm{PE}$ in diabetic rings was less in L-NAMEtreated diabetic rings $(-50 \%)$ compared to vehicle control-treated diabetic aortic rings $(-68 \%)$ (Table 1$)$.

\section{Discussion}

A major finding of the current work was that quercetin acutely increased ACh-induced endotheliumdependent relaxation, and reduced $\alpha_{1}$-adrenergic stimulant PE-induced contraction in diabetic rat aortic rings ex vivo, but did not have such effects in euglycemic rat aortic rings. In addition, L-NAME attenuated the vasodepressor effects of quercetin on PEinduced diabetic rat aortic ring contractions. Furthermore, quercetin reduced PE-induced contractions in LNAME-pretreated euglycemic rat aortic rings.
Previous studies have reported that various vascular preparations from diabetic animals show reduced relaxation in response to the endothelium-dependent and -independent vasodilators ACh and SNP, respectively, and enhanced contractions in response to the $\alpha_{1}$ adrenergic stimulant PE [3,18-20]. Increased production of free radicals including superoxide anions has been implicated in these processes [3-7]. Superoxide anions might inhibit endothelial relaxation or enhance receptor-mediated contractions either by enhancing vascular smooth muscle contraction or by inactivating endothelium-derived nitric oxide $[6,19,21,22]$.

Acute activation of endothelial nitric oxide synthase (eNOS) by $\mathrm{ACh}$ results in synthesis/release of endothelial nitric oxide, which in turn leads to guanyl cyclase activation, cGMP elevation and ultimately to vascular smooth muscle relaxation. In diabetic rat aortic rings, quercetin pretreatment increased ACh-induced relaxation but had no effect on the response to SNP. Therefore, the effect of quercetin on ACh-induced relaxation in diabetic rat aortic rings is unlikely to be due to a non-specific general improvement in vascular reactivity (e.g. activation of the endothelial nitric oxidecGMP relaxant pathway or enhanced cGMP production) $[19,23]$. The present data suggest quercetinenhanced ACh-induced relaxation in diabetic rat aortic rings was dependent on endothelium-derived nitric oxide. Recent data suggest the beneficial vascular effects of flavonoids are closely related to their free radical scavenging and antioxidant properties. Quercetin is a potent antioxidant and has been shown previously to protect nitric oxide from the scavenging actions of the superoxide anion [11,12,24,25]. Given 
that superoxide anions are involved in the reduced relaxation of diabetic arteries to ACh [3-7], the free radical scavenging action may explain the effects of quercetin on ACh-induced relaxation in diabetic rat aortic rings in the present study. We also found that whereas quercetin restored $\mathrm{ACh}$ responses in diabetic rat aortic rings, it had no effect on ACh responses in euglycemic rat aortic rings, where the production of superoxide anions has been thought to be too little to alter the bioavailability of endothelium-derived nitric oxide. This finding also suggests that the effects of quercetin were more pronounced when bioavailability of EDNO is impaired. Furthermore, quercetin restored ACh-induced relaxation in diabetic rat aortic rings to levels similar to those observed in euglycemic rat aortic rings. These observations further support our contention that quercetin improved $\mathrm{ACh}$ responses in diabetic rat aortic rings through its free radical scavenging actions.

The present results showed that quercetin had no effect on PE-induced contraction in euglycemic aortic rings, but significantly reduced in diabetic aortic rings. Quercetin caused a relaxation through endotheliumderived nitric oxide and prostacylin-pathways and/or decrease in intracellular calcium availability in smooth muscle cells in pre-contracted aortic rings isolated from euglycemic [13] and diabetic [15] rats. The present study involved the use of indomethacin, a cyclooxygenase inhibitor, in the organ chamber throughout the experiments, making it unlikely there was any relaxing effect from endothelium-derived prostacyclin or cross-talk between EDNO signaling pathways and prostanoids. In addition, the latter studies also reported that the pretreatment with quercetin was without any inhibitory effects on the contractions induced by PE in isolated euglycemic and diabetic rat aortic rings $[13,15]$. These results suggest that pretreatment with quercetin does not modify basal levels of endotheliumderived relaxant factors (EDRF) or modify calcium influx in both types of aortic rings. On the other hand, it is also reported that the vasoconstrictor effect of $\alpha$ adrenergic receptor agonists can be modulated by EDRF released by activation of $\alpha$-adrenoreceptor on the endothelial membrane [26]. Taking these previous reports into consideration, the present results indicate a possible involvement of free radical scavenging activity, i.e., protection of EDRF released by activation of endothelial $\alpha$-adrenoreceptor, in the quercetin attenuation of PE contraction of diabetic aortic rings. Two findings from the present study support this hypothesis. First, the vasodepressor effects of quercetin on PEinduced diabetic rat aortic ring contraction were attenuated by L-NAME, suggesting the quercetin effect was mediated, at least in part, by EDNO. Second, quercetin did not affect PE-induced contractions in euglycemic rat aortic rings.

The present study found that PE-induced contractions were lower in quercetin-pretreated diabetic rat aortic rings compared to euglycemic rat aortic rings. Furthermore, quercetin reduced PE responses in L-NAMEpretreated euglycemic and diabetic rat aortic rings. These findings that quercetin can act in the partial or complete absence of EDNO bioavailability suggest quercetin can exert its vasodepressor effects via mechanisms other than, or in addition to, those involving free radical scavenging and antioxidant protective effects on EDNO. Flavonoids have been recently reported to elicit endothelium-derived hyperpolarizing factor (EDHF)mediated vascular reactivity in vascular preparations $[27,28]$. The present experimental conditions inhibiting endothelial nitric oxide- and prostacyclin-mediated vasodilatation suggest the observed vasodepressor effect of quercetin may have involved EDHF. However, this assumption requires conformation. In addition, it is conceivable that the effects of quercetin would be much more pronounced when the bioavailability of EDNO is impaired since the attenuation by quercetin of $\mathrm{PE}$ contraction in rings from normal rats was not observed in the absence of L-NAME.

In summary, the present results show, for the first time, that quercetin restores endothelium-dependent vascular relaxation and reduces agonist-mediated constriction in aortas isolated from experimental diabetic rats. These beneficial actions of quercetin were mediated, at least in part, by its antioxidant protection of endothelium-derived nitric oxide. These acute effects of quercetin involving increased endothelium-dependent relaxation and reduced receptormediated contraction suggest mechanisms explaining the possible beneficial effects of quercetin in vivo in experimental diabetes and possibly in other cardiovascular diseases.

\section{Acknowledgement}

This study was supported by an IRPA grant (No.: 0602-03-6020) from Ministry of Science, Technology and Innovation, Malaysia.

\section{References}

[1] R.F. Furchgott, J.V. Zawadzki, The obligatory role of endothelial cells in the relaxation of arterial smooth muscle by acetylcholine, Nature 299 (1980) 373-376. 
[2] G.A. Rongen, P. Smits, T. Thien, Endothelium and the regulation of vascular tone with emphasis on the role of nitric oxide: physiology, pathophysiology and clinical implications, Neth. J. Med. 44 (1994) 26-35.

[3] D.W. Laight, M.J. Carrier, E.E. Anggard, Antioxidants, diabetes and endothelial dysfunction, Cardiovasc. Res. 47 (2000) 457-464.

[4] J.W. Baynes, Role of oxidative stress in development of complications in diabetes, Diabetes 40 (1991) 405-412.

[5] M.G. Cinar, S. Ulker, G. Alper, A. Evinc, Effect of dietary vitamin E supplementation on vascular reactivity of thoracic aorta in streptozotocin-diabetic rats, Pharmacology 62 (2001) 56-64.

[6] S. Dhein, A. Kabat, A. Olbrich, P. Rosen, H. Schroder, F.W. Mohr, Effect of chronic treatment with vitamin $\mathrm{E}$ on endothelial dysfunction in a type $I$ in vivo diabetes mellitus model and in vitro, J. Pharmacol. Exp. Ther. 305 (2003) 114-122.

[7] N.R.F. Nascimento, A. Costa-e-Forti, A.A. Peter, M.C. Fonteles, Free radical scavengers improve the impaired endotheliumdependent responses in aorta and kidneys of diabetic rabbits, Diab. Res. Clin. Pract. 61 (2003) 145-153.

[8] J.M. Geleijnse, L.J. Launer, D.A. Van der Kuip, A. Hofman, J.C. Witteman, Inverse association of tea and flavonoid intakes with incident myocardial infarction: the Rotterdam Study, Am. J. Clin. Nutr. 75 (2002) 880-886.

[9] W. Zenebe, O. Pechanova, I. Bernatova, Protective effects of red wine polyphenolic compounds on the cardiovascular system, Exp. Clin. Cardiol. 6 (2001) 153-158.

[10] R.J. Nijveldt, E. van Nood, D.E. van Hoorn, P.G. Boelens, K. van Norren, P.A. van Leeuwen, Flavonoids: a review of probable mechanisms of action and potential applications, Am. J. Clin. Nutr. 74 (2001) 418-425.

[11] C. Kandaswami, E. Middleton Jr., Free radical scavenging and antioxidant activity of plant flavonoids, Adv. Exp. Med. Biol. 366 (1994) 351-376.

[12] M. Ajay, H.M. Cheng, A.M. Mustafa, M.R. Mustafa, A comparative study on antioxidant activity of flavonoids: structureactivity relationships, Malaysian J. Sci. 24 (2005) 187-190.

[13] M. Ajay, A.H. Gilani, M.R. Mustafa, Effects of flavonoids on vascular smooth muscle of the isolated rat thoracic aorta, Life Sci. 74 (2003) 603-612.

[14] M. Vessal, M. Hemmati, M. Vasei, Antidiabetic effects of quercetin in streptozotocin-induced diabetic rats, Comp. Biochem. Physiol. C 135 (2003) 357-364.

[15] M. Roghani, T. Baluchnejadmojarad, M.R. Vaez-Mahdavi, F. Roghani-Dehkordi, Mechanisms underlying quercetin-induced vasorelaxation in aorta of subchronic diabetic rats: an in vitro study, Vasc. Pharmacol. 42 (2005) 31-35.
[16] M. Ajay, M.R. Mustafa, Chronic treatment with flavonoids prevents endothelial dysfunction in spontaneously hypertensive rat aorta, J. Cardiovasc. Pharmacol. 46 (2005) 36-40.

[17] J. Duarte, R. Perez-Palencia, F. Vargas, M.A. Ocete, F. PerezVizcaino, A. Zarzuelo, et al., Antihypertensive effects of the flavonoid quercetin in spontaneously hypertensive rats, Br. J. Pharmacol. 133 (2001) 117-124.

[18] L.S. Dresner, S.P. Wang, M.W. West, I.N. Ponomarenko, C.M. Mueller, R.B. Wait, Nitric oxide inhibition simulates the enhancement of $\alpha_{1}$-agonist-induced vasoconstriction in diabetes, J. Surg. Res. 70 (1997) 119-123.

[19] K. Kamata, N. Miyata, T. Abiru, Y. Kasuya, Functional changes in vascular smooth muscle and endothelium of arteries during diabetes mellitus, Life Sci. 50 (1992) 1379-1387.

[20] T. Baluchnejadmojarad, M. Roghani, Garlic extract attenuates time-dependent changes in the reactivity of isolated aorta in streptozotocin-diabetic rats, Life Sci. 73 (2003) 2281-2289.

[21] Z.S. Katusic, Superoxide anion and endothelial regulation of arterial tone, Free Radic. Biol. Med. 20 (1996) 443-448.

[22] G. Kodja, D. Harrison, Interactions between NO and reactive oxygen species: pathophysiological importance in atherosclerosis, hypertension, diabetes and heart failure, Cardiovasc. Res. 43 (1999) 557-562.

[23] K. Kamata, N. Miyata, Y. Kasuya, Impairment of endotheliumdependent relaxation and changes in levels of cyclic GMP in aorta from streptozotocin-induced diabetic rats, Br. J. Pharmacol. 97 (1989) 614-618.

[24] C. Yuting, Z. Rongliang, J. Zhongjian, J. Yong, Flavonoids as superoxide scavengers and antioxidants, Free Radic. Biol. Med. 9 (1990) 19-21.

[25] G. Lopez-Lopez, L. Moreno, A. Cogolludo, M. Galisteo, M. Ibaraa, J. Duarte, et al., Nitric oxide (NO) scavenging and NO protecting effects of quercetin and their biological significance in vascular smooth muscle, Mol. Pharmacol. 65 (2004) 851859.

[26] T. Godfraind, C. Egleme, A.I. Osachie, Role of endothelium in the contractile response of rat aorta to alpha-adrenergic agonists, Clin. Sci. 68 (10) (1985) 65s-71s.

[27] O.L. Woodman, M. Boujaoude, Chronic treatment of male rats with diadzein and 17-beta-oestradiol induces the contribution of EDHF to endothelium-dependent relaxation, Br. J. Pharmacol. 141 (2004) 322-328.

[28] R.S. De Moura, D.Z. Miranda, A.C. Pinto, R.F. Sicca, M.A. Souza, L.M. Rubenich, et al., Mechanism of the endotheliumdependent vasodilation and the antihypertensive effect of Brazilian red wine, J. Cardiovasc. Pharmacol. 44 (2004) 302-309. 